\title{
Micro and nano fabrication technologies for THz vacuum devices
}

\author{
Vishnu Srivastava
Microwave Tubes Division, CSIR-Central Electronics Engineering Research Institute, Pilani (Rajasthan) 333031, India
Email: vsceeri@gmail.com

Compact vacuum microelectronic devices (VMDs) are being investigated for efficient high power generation and amplification of terahertz (THz) frequencies from $0.1 \mathrm{THz}$ to $1 \mathrm{THz}$, as this frequency band has many applications. Due to unique properties of $\mathrm{THz}$ waves such as effective penetration through fabric and fog, non-ionizing radiation with reduced health risks, and molecular resonances that occur in this frequency band, $\mathrm{THz}$ technology is highly demanding for imaging, safety, defense, communication, remote sensing, astronomy and spectroscopy. $\mathrm{THz}$ waves provide high resolution images and are highly promising for medical diagnosis like skin cancer and tooth decay.

Development activities addressed to $0.22 \mathrm{THz}$ frequency band is of significant importance for medical imaging and communication because of the available atmospheric spectral window of $0.20 \mathrm{THz}$ to $0.30 \mathrm{THz}$. Present solid-state devices (SSDs) cannot be used at THz frequencies for output power more than few $\mu \mathrm{W}$. There are inherent advantages of vacuum electronic devices over SSDs in terms of higher output power, lower response time and higher efficiency because of electron beam movement in vacuum. Because of small size of rf components of vacuum devices at $\mathrm{THz}$ frequencies, micro-fabrication technologies like UV-LIGA and DRIE, are required for fabrication of these components. Therefore these vacuum devices are named as vacuum microelectronic devices (VMDs) [1] taking advantage of tube technology and solid-state (MEMS) technology. For such VMDs, field emitter arrays (FEAs) as shown in Figure 1, or carbon nano tubes (CNT) cold cathode [2], are used for generation of high current density electron beam. These cathodes may generate electron beam at room temperature with current density $10 \mathrm{~A} / \mathrm{cm}^{2}$ at low voltage. Also, sheet beam is used in place of round beam due to lower space charge force. It provides higher beam current capacity leading to higher efficiency and significant reduction of size and mass and operating beam voltage.

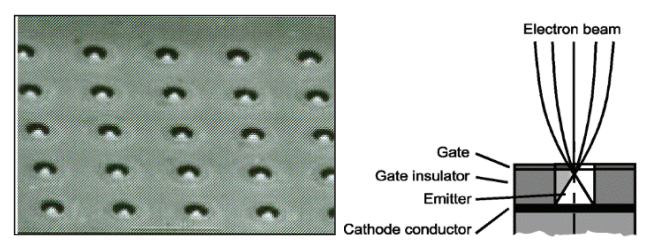

Figure 1: FEA cathode with electron beam emission
Among various VMDs, such as travelling wave tube (TWT), backward-wave oscillator (BWO), klystron, TWT is preferred as a high power $\mathrm{THz}$ amplifier due to its high efficiency over wide bandwidth and large thermal capacity. For medical imaging and communication, $0.22-\mathrm{THz}$ TWT of $10 \mathrm{~W}$ power, 20 $\mathrm{dB}$ gain, 5\% efficiency, and $30 \mathrm{GHz}$ bandwidth is being investigated. Planar slow-wave structure (SWS) [3], as shown in Figure 2, is selected for the proposed $0.22-\mathrm{THz}$ TWT because of its broad bandwidth and high impedance, and also it is easier to fabricate using MEMS technology with high accuracy and surface finish. Figure 2 shows 2D and 3D views of a halfperiod staggered double vane rectangular waveguide SWS with rectangular sheet beam. Dimensional parameters of the planar SWS for a $0.22-\mathrm{THz}$ TWT are given for $20 \mathrm{kV}$ electron beam: period (d) $460 \mu \mathrm{m}$, vane thickness (d-a) $110 \mu \mathrm{m}$, beam tunnel height (b) $150 \mu \mathrm{m}$, vane height (L) $270 \mu \mathrm{m}$, and circuit width (h) $770 \mu \mathrm{m}$.

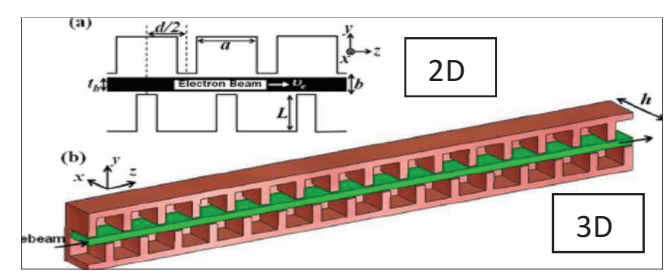

Figure 2: Planar slow-wave-structure for 0.22-THz TWT

Micro-fabrication technologies like UV-LIGA with SU-8 process are to be optimised for fabrication of the RF structure with high precision and surface finish. There are many criticalities in fabrication like dimensional tolerance within $\pm 2 \mu \mathrm{m}$, surface roughness $<10 \mathrm{~nm}$, alignment of beam hole in the assembled twohalves of the rf structure within $2 \mu \mathrm{m}$, tapered vane heights at input and output for matching of SWS with waveguide couplers, and incorporation of sever loss in between circuit. MEMS technologies available at CSIR-CEERI are being explored for fabrication of the RF structure and FEA cold cathode.

\section{References}

1. Vishnu Srivastava, $\mathrm{THz}$ vacuum microelectronic devices, Journal Physics, vol. 114, no. 1, 2007, pp. 12015-25.

2. Carter M Armstrong, The quest for the ultimate vacuum tube, IEEE Spectrum, Nov.2015, pp.30-40.

3. Anisullah Baig,et.al., MEMS compatible $220 \mathrm{GHz}$ TWTA, Progress in Electromagnetics, vol. 41, 2013, pp.13548. 